\title{
Time-resolved detection of propagating Lamb waves in thin silicon membranes with frequencies up to $197 \mathrm{GHz}$
}

\author{
Martin Grossmann, ${ }^{1, a)}$ Oliver Ristow, ${ }^{1}$ Mike Hettich, ${ }^{1}$ Chuan He, ${ }^{1}$ Reimar Waitz, ${ }^{1}$ \\ Elke Scheer, ${ }^{1}$ Vitalyi Gusev, ${ }^{2}$ Thomas Dekorsy, ${ }^{1}$ and Martin Schubert ${ }^{1}$ \\ ${ }^{1}$ Department of Physics, University of Konstanz, 78457 Konstanz, Germany \\ ${ }^{2}$ LUNAM Universités, CNRS, Université du Maine, LAUM UMR-CNRS 6613, Av. O. Messiaen, \\ 72085 Le Mans, France
}

(Received 6 February 2015; accepted 16 April 2015; published online 27 April 2015)

\begin{abstract}
Guided acoustic waves are generated in nanopatterned silicon membranes with aluminum gratings by optical excitation with a femtosecond laser. The spatial modulation of the photoacoustic excitation leads to Lamb waves with wavelengths determined by the grating period. The excited Lamb waves are optically detected for different grating periods and at distances up to several $\mu \mathrm{m}$ between pump and probe spot. The measured frequencies are compared to the theoretical dispersion relation for Lamb waves in thin silicon membranes. Compared to surface acoustic waves in bulk silicon twice higher frequencies for Lamb waves (197 GHz with a $100 \mathrm{~nm}$ grating) are generated in a membrane at equal grating periods. (C) 2015 AIP Publishing LLC.
\end{abstract}

[http://dx.doi.org/10.1063/1.4919132]

Lamb waves are acoustic waves guided between the mechanically free opposite surfaces of an elastic plate. Various methods for the generation and detection of Lamb waves by lasers have been developed for applications in noncontact nondestructive testing, in evaluation of thin films, and in free-standing plates. ${ }^{1-5}$ These investigations include the all-optical monitoring of zero-group-velocity Lamb modes as demonstrated recently by Prada et $a l^{4,5}$ Continuous progress in the development of methods for the fabrication of membranes with a thickness in the nm range for a variety of applications ${ }^{6}$ requires the evaluation of their parameters and quality, e.g., their dispersion relation and damping, the development of methods for both the generation and detection of acoustic waves at $\mathrm{GHz}$ frequencies.

Standing waves in free-standing films of $\mu \mathrm{m}$ thickness have been first detected by Brillouin scattering. ${ }^{7}$ Recently, femtosecond lasers have been successfully applied to generate and detect longitudinal acoustic modes in free-standing silicon $(\mathrm{Si})$ membranes with frequencies up to $500 \mathrm{GHz}$ corresponding to $20 \mathrm{~nm}$ wavelengths. ${ }^{8-10}$ As the dispersion relations of propagating Lamb waves are emerging from standing wave resonances, ${ }^{11}$ these experiments indicate that propagating Lamb waves in membranes at similarly high frequencies could be monitored all-optically as well, if the conditions for their effective generation and detection are achieved. Recently, Rayleigh waves with wavelengths down to $45 \mathrm{~nm}$ (Refs. 12 and 13) and frequencies up to $90 \mathrm{GHz}$ (Ref. 12) have been generated and detected based on the deposition of a metallic grating of nanometer periodicity on the substrate surface. Rayleigh waves are surface acoustic waves on a semi-infinite substrate. ${ }^{14}$ Here, we combine the femtosecond pump-probe technique applied earlier to the monitoring of standing wave resonances ${ }^{8-10}$ and of Rayleigh waves $^{12}$ with nanostructuring of the surface ${ }^{12,13,15}$ in order to achieve the generation and detection of propagating Lamb

a)martin.grossmann@uni-konstanz.de waves at frequencies up to $197 \mathrm{GHz}$ and wavelengths down to $50 \mathrm{~nm}$ in thin semiconductor membranes. Those high frequencies can be generated because for the same wave vectors the dispersion relations of Lamb waves contain modes of much higher frequencies than those of Rayleigh waves. The highest frequencies of the detected coherent Lamb modes are nearly three times higher than the highest frequencies of thermal Lamb modes detected by classical Brillouin scattering. ${ }^{16}$

Different samples with aluminum $(\mathrm{Al})$ gratings from $100 \mathrm{~nm}$ to $500 \mathrm{~nm}$ period are prepared on crystalline Si membranes $(326 \mathrm{~nm}$ thickness). The grating stripes are oriented perpendicular to the [110]-direction on the (001)-surface of $\mathrm{Si}$ with a filling factor of $50 \%$. The thickness of the $\mathrm{Al}$ is $17 \mathrm{~nm}$ and the stripes are partly embedded in the membrane (see Fig. 1). A part of the membrane is covered by a uniform Al film for reference measurements. Details regarding the fabrication of the membranes and the gratings are discussed in the previous publications. ${ }^{12,17}$

The method and setup used are similar to the one previously employed for the generation and detection of Rayleigh waves. ${ }^{12}$ We excite and detect the Lamb waves with an

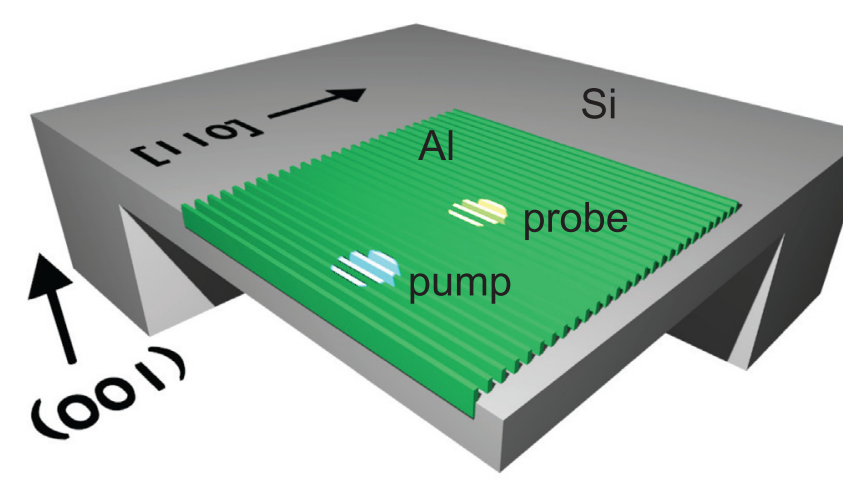

FIG. 1. Pump-probe scheme of the measurement on a thin Si membrane with an $\mathrm{Al}$ grating. Pump and probe pulses are laterally displaced. 
optical pump-probe technique based on high-speed asynchronous optical sampling (ASOPS) with femtosecond lasers at $\mathrm{GHz}$ repetition rate. ${ }^{18}$ The measurements are performed in a confocal microscope configuration, in which the laser spots can be focussed below $2 \mu \mathrm{m}$ full width at half maximum (FWHM) on the sample surface. The pump (probe) polarization is perpendicular (parallel) to the grating stripes. For measurements of the propagation of the Lamb waves, pump and probe spots can be spatially displaced relative to each other in the focal plane. The wavelengths for the pump and probe are $790 \mathrm{~nm}$ with a power of $20 \mathrm{~mW}$ and $820 \mathrm{~nm}$ with a power of $3.5 \mathrm{~mW}$, respectively. For the single stripe measurement, one and a half and four times larger intensities were used in the pump and the probe laser beams, respectively.

Fig. 2 shows three different temporal transients for a grating with $100 \mathrm{~nm}$ period, corresponding to three different distances between pump and probe spots. The top graph shows the measurement with spatially overlapping pump and probe spots. A strong change in reflectivity can be seen in the beginning of the transient, marking the time at which the pump pulse excites electron-hole pairs in the Si membrane. Superimposed on the decay of the electronic contribution are oscillations, which are shown in the inset. The background is subtracted in order to improve the visibility of the oscillatory components. These are coherently excited acoustic phonons representing acoustic eigenmodes of the membrane. The middle and bottom graphs show two measurements with distances of $6.8 \mu \mathrm{m}$ and $7.8 \mu \mathrm{m}$ between the pump and probe spots. The oscillation maxima (the center of the wavepackets indicated by the black arrows) are centered around $300 \mathrm{ps}$ for the shorter and $800 \mathrm{ps}$ for the longer distances. The temporal difference between the two maxima reflects the additional propagation length, which the acoustic wave packet needs to travel in the membrane from the pump to the probe spot. The $1 \mu \mathrm{m}$ larger distance results in 500 ps delay,

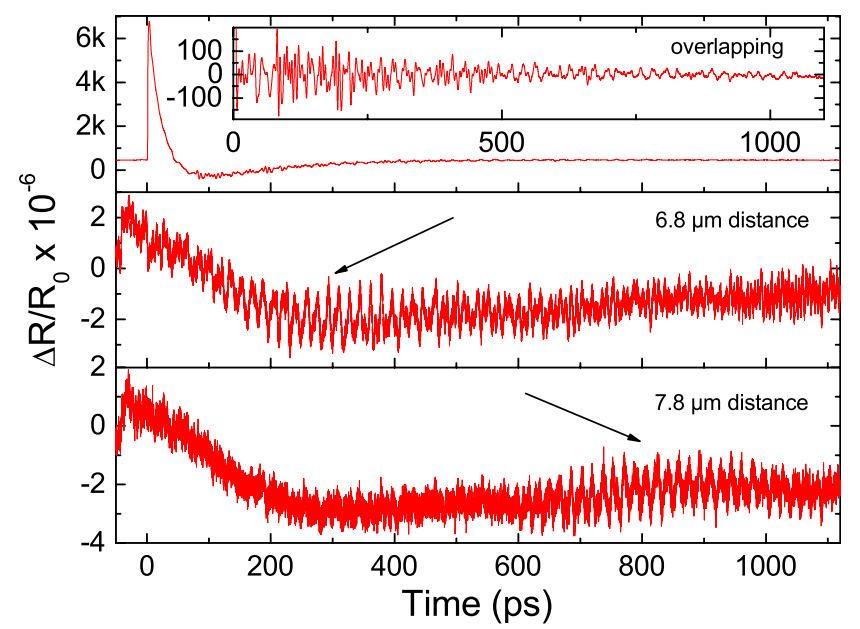

FIG. 2. Time-resolved modulation of the reflected probe beam $\Delta R / R_{0}$ of the $326 \mathrm{~nm}$ Si membrane with Al grating ( $17 \mathrm{~nm}$ thickness and $100 \mathrm{~nm}$ period) on top. The transient at the top shows the modulation when pump and probe spots are overlapped on the grating. The transient in the middle (bottom) shows the modulation when pump and probe are separated by $6.8 \mu \mathrm{m}$ $(7.8 \mu \mathrm{m})$. The arrows indicate the position of the center of the individual wavepackets. Note a change in the amplitude of $\Delta R / R_{0}$ by three orders of magnitude between top and middle/bottom graphs. which corresponds to a group velocity of around $2000 \mathrm{~m} / \mathrm{s}$. The acoustic wave packet would need for the $6.8 \mu \mathrm{m}$ distance, $3.4 \mathrm{~ns}$. We can therefore conclude that due to the high repetition rate of the lasers $(800 \mathrm{MHz})$, the measured signal stems from a preceding pump pulse at a multiple of the inverse repetition rate $(1.25 \mathrm{~ns})$. The propagating Lamb modes are discussed in more detail below.

In Figs. 3(a)-3(c), the Fourier spectra for the $100 \mathrm{~nm}$ grating corresponding to the time-domain data in Fig. 2 are shown. The spectrum where both pump and probe are spatially overlapping (a) shows a dense frequency distribution. The two spectra ((b) and (c)), taken at a distance of 6.8 and $7.8 \mu \mathrm{m}$ between pump and probe, show a strong change of the amplitude ratios of different spectral components and a less dense mode spectrum; a total of sixteen modes can be clearly resolved. As previously observed for Rayleigh waves, we assume that the grating structure excites wavevectors corresponding to the inverse grating period. ${ }^{12}$ Additionally, the Fourier analysis reveals that odd spatial harmonics are excited due to the fact that the grating is not sinusoidal but rectangular, while even spatial harmonics can be excited because of defects in the grating periodicity.

The observed frequencies are plotted in Fig. 4 for two different wave vectors, i.e., their corresponding grating period: the basic grating period and the first even harmonic (black diamonds). Further, we plot the Lamb dispersion relation for a thin Si plate along the [110]-direction. ${ }^{19}$ By looking at the basic wave vector, most of the measured frequencies can be clearly assigned to a mode given by the Lamb dispersion. Slight discrepancies can be explained by the measurement system: the sample is excited every $1.25 \mathrm{~ns}$ with $800 \mathrm{MHz}$, which limits the frequency resolution to around $1 \mathrm{GHz}$ and also limits the determination of the wavevector due to the finite length of the wave packet excited with the grating; the grating is illuminated with a pump spot of less than $2 \mu \mathrm{m}$ FWHM. When the grating period gets smaller, the error due to the finite laser spot decreases. As well, the fabrication process gives both a variation in grating period and membrane thickness $( \pm 5 \mathrm{~nm}$ ), leading to an increased error for smaller grating periods. Both

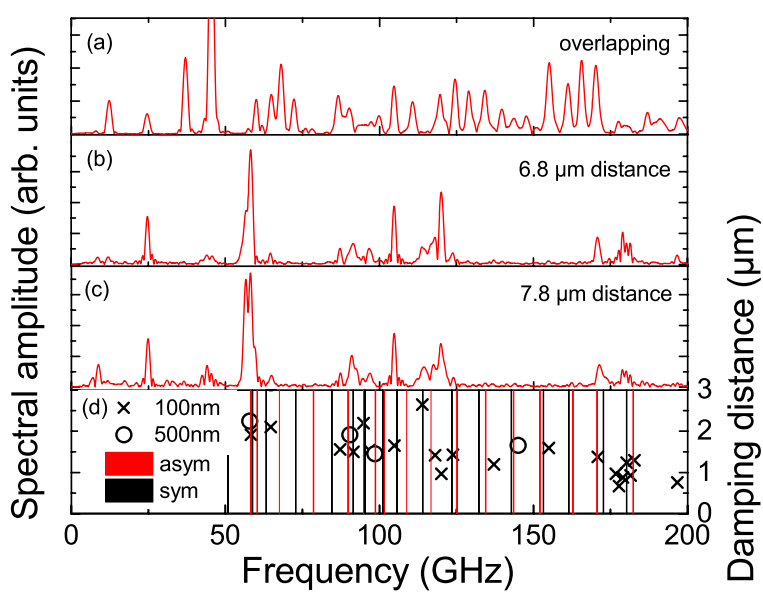

FIG. 3. (a)-(c) Fourier spectra of the different time transients in Fig. 2. (d) The damping distance over frequency is plotted for the $100 \mathrm{~nm}$ and the $500 \mathrm{~nm}$ grating and the expected frequencies given by the Lamb dispersion relation are shown by vertical lines (for symmetric modes in red and for antisymmetric in black). 


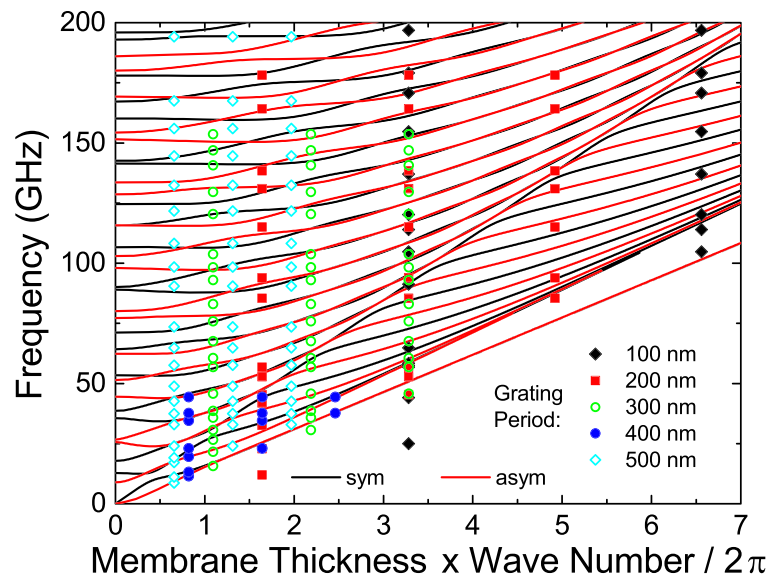

FIG. 4. Dispersion relation of Lamb modes in a thin Si membrane in the [110]-direction. Black lines mark the symmetric modes and red lines mark the asymmetric ones. The data points correspond to the first, second, and third harmonic of the grating period (left to right) and the corresponding measured frequencies (see Fig. 3).

contributions lead to a nearly homogeneous error of 5\%-6\% for the wavevector of every grating period.

There are some frequencies, however, that cannot be attributed to a frequency given by the dispersion relation. Here, the higher harmonics are important. We checked, for example, that the experimental frequency of $196.8 \mathrm{GHz}$ is closer to the theoretical frequency of $196.3 \mathrm{GHz}$ of the 15 th asymmetric Lamb mode with the second harmonic of the grating period than to the frequency of $192.1 \mathrm{GHz}$ of the 19th symmetric Lamb mode with the basic grating period. The second harmonic therefore means that this mode has a wavelength of $50 \mathrm{~nm}$.

In the spectra with the spatially separated pump and probe, the $58.4 \mathrm{GHz}$ mode has the largest amplitude. It corresponds to a Lamb wave of $100 \mathrm{~nm}$ wavelength with a phase velocity of about $5840 \mathrm{~m} / \mathrm{s}$, which is close to the transversal speed of sound in bulk Si. We attribute the large amplitude to the fact that in the dispersion relation, two Lamb modes are close together in the vicinity of this frequency and wavevector.

There are two modes which cannot be explained by any of the harmonics: at 24.9 and $44.1 \mathrm{GHz}$. Due to the spatial width of the pump laser spot we also excite modes with a small wavevector, quite smaller than for the grating period itself. As can be seen in Fig. 4, two modes can be found at small wave vectors that match the measured frequencies very well. A priori we cannot distinguish any of the measured frequencies by their excitation processes (finite spot size, grating period, and higher harmonic). However, as explained above, for some of the individual frequencies we are sure that they correspond to higher harmonics of the grating period.

Figure 3(d) shows the damping for the individual modes. The damping distance, i.e., the distance over which the amplitude reaches 1/e, was evaluated by fitting the FFT amplitudes with an exponential decay over distance. For the lower frequencies, damping distances around $2 \mu \mathrm{m}$ are found, while for the higher frequencies lower damping distances around $1 \mu \mathrm{m}$ are found.

We perform the same experiments on samples with grating periods of 200 to $500 \mathrm{~nm}$ in $100 \mathrm{~nm}$ steps. Up to 21 modes are visible. All observed frequencies are plotted in Fig. 4 with respect to their grating period including the first three harmonics. Because it is not possible to distinguish, which mode belongs to which wavevector, we did not assign any mode to an individual wavevector. As for the $100 \mathrm{~nm}$ grating case, most of the measured frequencies can be clearly assigned to a frequency given by the Lamb dispersion. The damping distance for the $500 \mathrm{~nm}$ grating is shown in Fig. 3(d) together with the $100 \mathrm{~nm}$ case. For both structures, a similar frequency dependent damping behavior can be seen.

The measured Lamb modes are excited due to two different physical mechanisms: one is the thermo-elastically generated stress in the $\mathrm{Al}$ grating, leading to a local negative strain, and the other one is due to the deformation potential in $\mathrm{Si}$, leading to a positive strain. Both effects need to be taken into consideration. The periodic expansion of the $\mathrm{Al}$ metal stripes on top of the grating as well as the compression of the underlying membrane, which is being masked for optical excitation by the grating on top, ${ }^{20}$ also lead to a periodic modulation. To calculate both contributions is beyond the scope of this article, as not only the strain is difficult to calculate due to carrier diffusion ${ }^{21,22}$ but also the optical field that excites the structure. ${ }^{23,24}$

We attribute the absence of some expected modes to the mutual compensation of two different contributions to the detection process: Two physical mechanisms play a role in the detection of the Lamb mode. These are the photoelastic effect in $\mathrm{Al}$ and $\mathrm{Si}$ and the interferometric effect in the $\mathrm{Si}$ membrane. The interferometric effect is caused by surface corrugation due to the Lamb waves, i.e., the relative motion of the opposite surfaces, which causes modification of the membrane thickness. Quite recently, it has been demonstrated $^{25}$ that the optical detection process of confined eigenmodes can be either enhanced or reduced to zero following a selection rule. In particular, it has been shown that detection configurations exist where the coherent acoustic phonons and probe light sensitivity functions are orthogonal, leading to cancelation of the photoelastic detection mechanism.

We investigate the detection process more closely with a different sample structure. The excitation was performed on two (nominal) $100 \mathrm{~nm}$ gratings on the same wafers (A1 and A2). We first compared these nominally identical gratings by detecting the spectra of their vibrations with the pump and probe beams overlapping on the gratings (see top panel in Fig. 5). This comparison demonstrates that the main spectral characteristics are well reproduced especially at frequencies of propagating Lamb modes (above $50 \mathrm{GHz}$ ). Then, we detected the Lamb spectra in the case where the pump and probe beams are spatially separated. In sample A1, the Lamb waves were propagating all their path of $6.8 \mu \mathrm{m}$ along the grating, while in the sample A2 they propagated $5 \mu \mathrm{m}$ along the grating and $10 \mu \mathrm{m}$ along the free surface of the $\mathrm{Si}$ membrane between the edge of the grating and a single detecting stripe (width: $1.5 \mu \mathrm{m}$ ). The comparison of the detected spectra in the bottom panel of Fig. 5 indicates that the metallic grating enhances the optical detection especially of high frequency Lamb modes. 


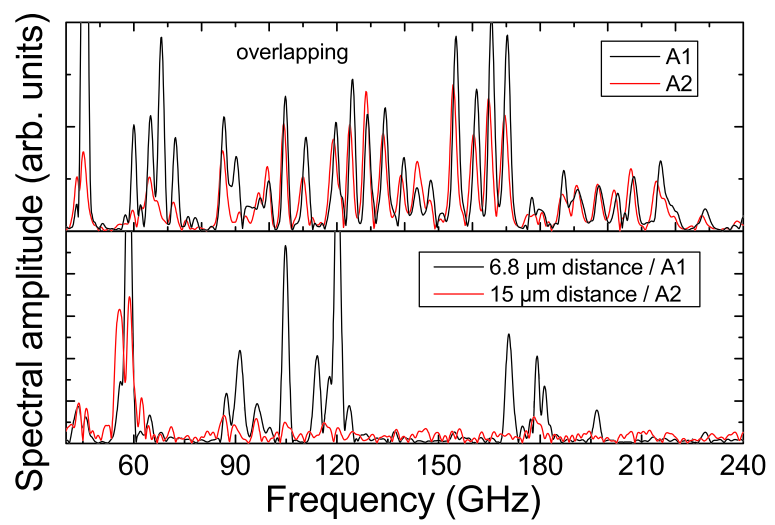

FIG. 5. Fourier spectra of two measurements where the pump is on two different $100 \mathrm{~nm}$ gratings. The top measurement was done with pump and probe overlapped for two different samples (A1 and A2) with nominally the same properties. The bottom spectra are taken at a distance between pump and probe spots with the pump spot on the grating. The probe spot is on the grating in $6.8 \mu \mathrm{m}$ distance (A1) or on a $1.5 \mu \mathrm{m}$ wide single stripe deposited in $10 \mu \mathrm{m}$ distance parallel to the grating (A2) with $15 \mu \mathrm{m}$ distance to the pump spot.

One also has to keep in mind that the Al grating may not only allow to excite ultra-high frequency modes but also affect the eigenmodes of the membrane. Although the difference in longitudinal impedances of $\mathrm{Al}$ and $\mathrm{Si}$ is negligible, the difference in shear impedances is significant and, thus, the modification of the spectra of the Lamb modes in the presence of the $\mathrm{Al}$ grating is to be expected. However, it has been demonstrated earlier ${ }^{12}$ that even for the Rayleigh waves of $100 \mathrm{~nm}$ length localized within $100 \mathrm{~nm}$ from the surface covered by a similar $\mathrm{Al}$ grating, the frequency shift does not exceed $10 \%$. The higher order Lamb modes, guided by the complete thickness of the membrane $(324 \mathrm{~nm})$, that are overlapping with the fingers are more than three times weaker than that. Thus, we would expect that the frequency modification caused by the grating does not exceed $5 \%-6 \%$. These effects should be taken into account if a precise identification of the Lamb modes is necessary.

We showed that thin Si membranes represent an excellent system to generate guided waves in the hypersonic frequency range. For the same wavelength, higher-order Lamb modes are of higher frequency in comparison with Rayleigh waves. This provides the opportunity to generate higher frequencies by laser-excited structures with the same spatial modulation. We were able to generate frequencies up to $197 \mathrm{GHz}$ with wavelengths down to $50 \mathrm{~nm}$.

There is currently growing interest to the application of the resonances of guided acoustic waves in nanocantilevers for the evaluation of liquids, ${ }^{26,27}$ to the interaction of Rayleigh surface acoustic waves with other types of excitations, and also with charge carriers in the vicinity of the solid surface (see Refs. 28-30 and the references therein). Thus, we can reasonably expect that Lamb waves of hundreds of $\mathrm{GHz}$ frequencies and tens of nanometers wavelengths would find applications not only in the testing and evaluation of thin membranes but also for studying the fundamental problems of liquid rheology at $\mathrm{GHz}$ frequencies through experiments with liquid-loaded membranes and for probing the interaction of travelling acoustic waves with charge carriers and other type of excitations in solids in nanometer-confined geometries. 31

This work was supported by the DFG through the SFB 767 (Germany) and by the Ministry of Science, Research, and Arts of Baden-Württemberg (Germany). The authors also thank Matthias Hagner for providing support in the nanostructure laboratory.

${ }^{1}$ C. B. Scruby and L. E. Drain, Laser Ultrasonics, Techniques and Applications (Taylor \& Francis Group, New York, 1990).

${ }^{2}$ J. S. Meth, C. D. Marshall, and M. D. Fayer, J. Appl. Phys. 67, 3362 (1990).

${ }^{3}$ J. A. Rogers, A. A. Maznev, M. J. Banet, and K. A. Nelson, Annu. Rev. Mater. Sci. 30, 117 (2000).

${ }^{4}$ C. Prada, O. Balogun, and T. W. Murray, Appl. Phys. Lett. 87, 194109 (2005).

${ }^{5}$ C. Prada, D. Clorennec, and D. Royer, J. Acoust. Soc. Am. 124, 203 (2008).

${ }^{6}$ J. A. Rogers, M. G. Lagally, and R. G. Nuzzo, Nature 477, 45 (2011).

${ }^{7}$ J. Sandercock, Phys. Rev. Lett. 29, 1735 (1972).

${ }^{8}$ F. Hudert, A. Bruchhausen, D. Issenmann, O. Schecker, R. Waitz, A. Erbe, E. Scheer, T. Dekorsy, A. Mlayah, and J.-R. Huntzinger, Phys. Rev. B 79, 201307 (2009).

${ }^{9}$ A. Bruchhausen, R. Gebs, F. Hudert, D. Issenmann, G. Klatt, A. Bartels, O. Schecker, R. Waitz, A. Erbe, E. Scheer, J.-R. Huntzinger, A. Mlayah, and T. Dekorsy, Phys. Rev. Lett. 106, 077401 (2011).

${ }^{10}$ J. Cuffe, O. Ristow, E. Chávez, A. Shchepetov, P.-O. Chapuis, F. Alzina, M. Hettich, M. Prunnila, J. Ahopelto, T. Dekorsy, and C. M. SotomayorTorres, Phys. Rev. Lett. 110, 095503 (2013).

${ }^{11}$ B. A. Auld, Acoustic Fields and Waves in Solids, 2nd ed. (Krieger, Malabar, 1990), Vol. II.

${ }^{12}$ M. Schubert, M. Grossmann, O. Ristow, M. Hettich, A. Bruchhausen, E. C. S. Barretto, E. Scheer, V. Gusev, and T. Dekorsy, Appl. Phys. Lett. 101, 013108 (2012).

${ }^{13}$ Q. Li, K. Hoogeboom-Pot, D. Nardi, M. M. Murnane, H. C. Kapteyn, M. E. Siemens, E. H. Anderson, O. Hellwig, E. Dobisz, B. Gurney, R. Yang, and K. a. Nelson, Phys. Rev. B 85, 195431 (2012).

${ }^{14}$ L. Rayleigh, Proc. London Math. Soc. s1-17, 4 (1885).

${ }^{15}$ B. Bonello, A. Ajinou, V. Richard, P. Djemia, and S. M. Cherif, J. Acoust. Soc. Am. 110, 1943 (2001).

${ }^{16}$ M. Grimsditch, R. Bhadra, and I. Schuller, Phys. Rev. Lett. 58, 1216 (1987).

${ }^{17}$ M. Grossmann, M. Klingele, P. Scheel, O. Ristow, M. Hettich, C. He, R. Waitz, M. Schubert, A. Bruchhausen, V. Gusev, E. Scheer, and T. Dekorsy, Phys. Rev. B 88, 205202 (2013).

${ }^{18}$ A. Bartels, R. Cerna, C. Kistner, A. Thoma, F. Hudert, C. Janke, and T. Dekorsy, Rev. Sci. Instrum. 78, 035107 (2007).

${ }^{19}$ L. G. Merkulov and D. A. Tursunov, Sov. Phys. - Acoust. 15, 115 (1969).

${ }^{20} \mathrm{G}$. Antonelli, P. Zannitto, and H. J. Maris, Physica B 316-317, 377 (2002).

${ }^{21}$ G. Tas and H. Maris, Phys. Rev. B 49, 15046 (1994).

${ }^{22}$ O. Wright, B. Perrin, O. Matsuda, and V. Gusev, Phys. Rev. B 64, 081202 (2001).

${ }^{23}$ A. Barbara, P. Quémerais, E. Bustarret, and T. Lopez-Rios, Phys. Rev. B 66, 161403 (2002).

${ }^{24}$ J. Steele, C. Moran, A. Lee, C. Aguirre, and N. Halas, Phys. Rev. B 68, 205103 (2003).

${ }^{25}$ C. Mechri, P. Ruello, and V. Gusev, New J. Phys. 14, 023048 (2012).

${ }^{26}$ S. S. Verbridge, L. M. Bellan, J. M. Parpia, and H. G. Craighead, Nano Lett. 6, 2109 (2006).

${ }^{27}$ D. M. Karabacak, V. Yakhot, and K. L. Ekinci, Phys. Rev. Lett. 98, 254505 (2007).

${ }^{28}$ E. A. Cerda-Méndez, D. N. Krizhanovskii, M. Wouters, R. Bradley, K. Biermann, K. Guda, R. Hey, P. V. Santos, D. Sarkar, and M. S. Skolnick, Phys. Rev. Lett. 105, 116402 (2010).

${ }^{29}$ H. Sanada, T. Sogawa, H. Gotoh, K. Onomitsu, M. Kohda, J. Nitta, and P. V. Santos, Phys. Rev. Lett. 106, 216602 (2011).

${ }^{30}$ S. Büyükköse, B. Vratzov, J. van der Veen, P. V. Santos, and W. G. van der Wiel, Appl. Phys. Lett. 102, 013112 (2013).

${ }^{31}$ T. Dehoux, M. A. Ghanem, and O. F. Zouani, Ultrasonics 56, 160 (2015). 